\title{
LES MODIFICATIONS DES MLIEUX AQUATIQUES DE CAMARgUE AU COURS DES 30 DERNIÈRES ANNEES
}

\author{
par P. Aguesse et F. Marazanof.
}

Le but de la présente note n'est pas de reprendre en détail tous les travaux écologiques déjà publiés sur la Camargue, mais d'essayer de tirer, grâce à ces documents et à nos observations depuis 1953 . des conclusions plus générales sur l'évolution des milieux aquatiques depuis les 30 dernières années.

Pour cette étude, nous nous sommes inspirés des documents de : J. Blondel [1964], H. Engel [1957], L. Hertzog [1935], P. HeurTeaux [1962,] L. Hoffmann [1959, 1962], M. Paulus [1949,] G. Petit et D. Schachter $\lfloor 1954\rfloor$, D. Schachter $[1944,1945,1950$, 1960], D. Schachter et M. Conat 1951$]$, G. Tallon $[1938,1949$, $1950,1954]$ et de nos observations depuis 1953 : P. Aguesse [1955, $1956,1957,1958,1960,1961]$, P. Aguesse et L. Bigot $[1959,1960$, $1962]$, P. Aguesse et B. Dussart [1956], F. Marazanof [1963, 1964, 1965], Nourisson et P. Aguesse [1961].

Les données extraites de ces documents ne permettent d'avoir, avant 1953, que des données très fragmentaires floristiques et faunistiques sur quelques marais. A partir de 1953, nous avons pu étendre régulièrement nos prospections qualitatives et quantitatives dans le delta du Rhône.

L'extension de la riziculture dans la partie nord de la Camargue et la portion comprise à l'est et à l'ouest de la moyenne Camargue près des bras du Rhône (19000 ha de rizières en 1962), l'augmentation dans la partie sud-ouest de la zone des salins de la Compagnie Salicam ont changé, au cours des 30 dernières années la physionomie de la Camargue et créé un nouveau complexe hydrographique.

C'est grâce à nos relevés réguliers dans ces différentes zones et dans la «zone saumâtre » proprement dite du delta qu'il nous est aujourd'hui possible de tirer des conclusions générales de cette évolution, et des transformations des différents biotopes. Plus encore, ces données nous permettent de prévoir les répercussions qu'auraient d'éventuelles modifications du milieu sur la flore et la faune aquatique. 


\section{I. - DONNÊES Clmatiques}

Bien que maintes fois décrites, ces données nous paraissent essentielles pour expliquer le régime hydrique de la Camargue. Trois traits caractéristiques sont à retenir :

- étés chauds et secs, hivers relativement doux,

- pluies abondantes, au printemps et surtout en automne,

- fréquence importante des vents, en particulier du mistral qui accélère l'évaporation, intense dans cette région.

Données climatiques 1944-1961

\begin{tabular}{|c|c|c||}
\hline & Moyennes mensuelles & $\begin{array}{c}\text { Moyennes } \\
\text { précipitations (mm) }\end{array}$ \\
\cline { 2 - 3 } & & \\
\hline Janvier & 6,0 & 39,3 \\
Février & 7,7 & 30,8 \\
Mars & 10,8 & 48,3 \\
Avril & 13,8 & 38,2 \\
Mai & 17,1 & 43,7 \\
Juin & 20,7 & 24,0 \\
Juillet & 23,3 & 11,2 \\
Août & 22,6 & 30,2 \\
Septembre & 20,1 & 69,1 \\
Octobre & 15,3 & $\mathbf{9 3 , 9}$ \\
Novembre & $\mathbf{1 0 , 5}$ & 63,2 \\
Décembre & $\mathbf{7 , 5}$ & 69,0 \\
\hline
\end{tabular}

\begin{tabular}{|c|c|c||}
\hline & Précipitations $(\mathrm{mm})$ & $\begin{array}{c}\text { Nombre de jours } \\
\text { de pluie }\end{array}$ \\
\cline { 1 - 3 } & & \\
\hline 1944 & 387,6 & 56 \\
1945 & 320,4 & 51 \\
1946 & 399,9 & 64 \\
1948 & 505,2 & 65 \\
1949 & 556,9 & 59 \\
1950 & 515,5 & 61 \\
1951 & 429,4 & 52 \\
1952 & 35,4 & 88 \\
1953 & 999,8 & 58 \\
1954 & 903,6 & 70 \\
1955 & 525,7 & 75 \\
1956 & 698,0 & 73 \\
1957 & 732,2 & 59 \\
1958 & 559,7 & 67 \\
1959 & 405,8 & 66 \\
1960 & 665,8 & 91 \\
1961 & 715,6 & 64 \\
1962 & 510,5 & 77 \\
1963 & 417,4 & 99 \\
\hline
\end{tabular}




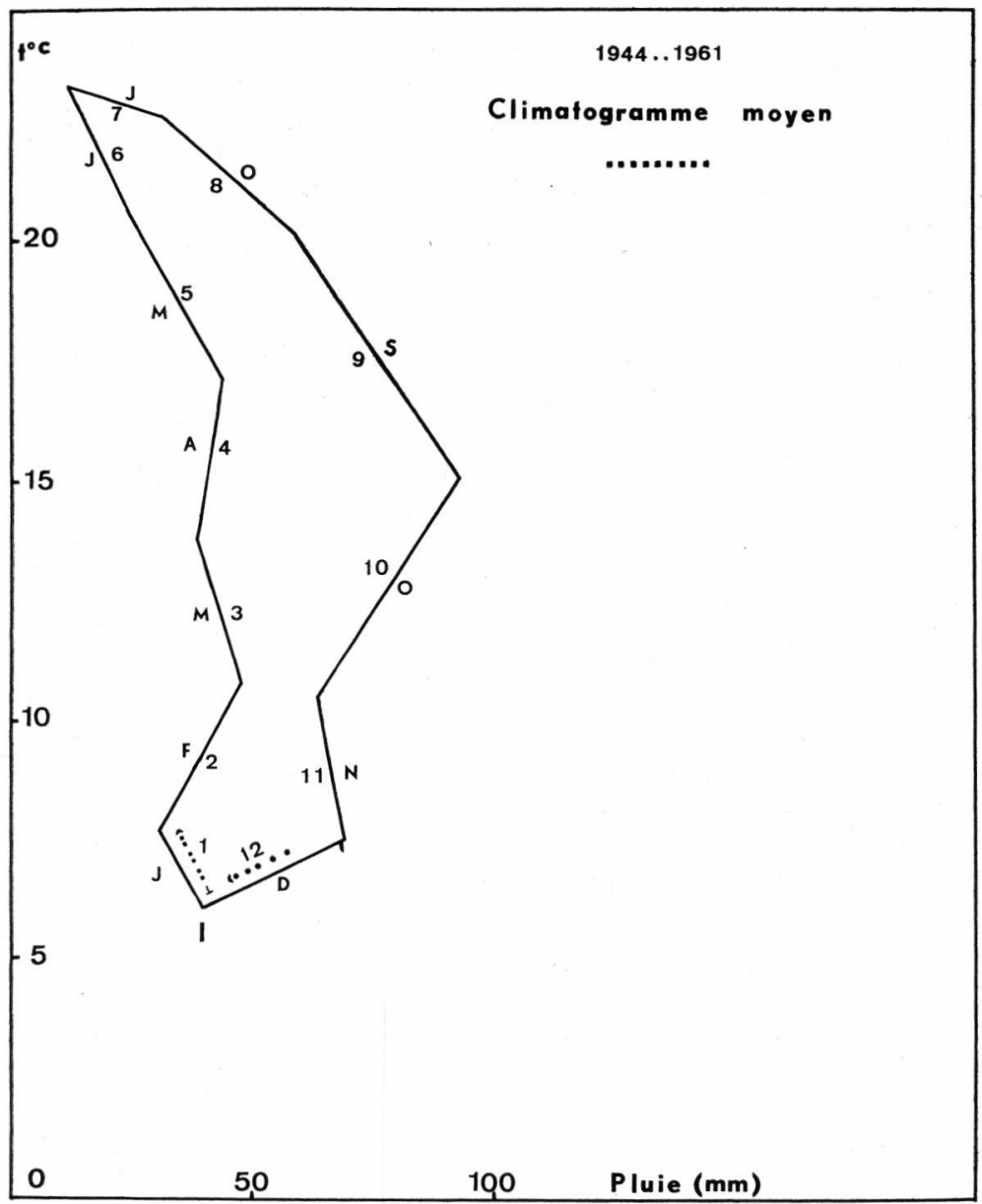

FIG. 1. Climatogramme moyen des années 1944 à 1961. Les chiffres $1,2,3_{*}$ 4 ... indiquent les différents mois de l'année.

La moyenne des précipitations annuelles établie de 1944-1961 est de $560,9 \mathrm{~mm}$ environ. Ces valeurs varient d'une année à l'autre ; ainsi il est tombé, en 1962, 417,4 mm (année sèche).

1962 : Précipitations (mm).

$\frac{\text { J. }}{29,8}\left|\frac{\text { F. }}{53,3}\right| \frac{\text { M. }}{16,5}\left|\frac{\text { A. }}{42,6}\right| \frac{\text { M. }}{8,4}\left|\frac{\text { J. }}{28,8}\right| \frac{\text { J. }}{0,9}\left|\frac{\text { A. }}{1,7}\right| \frac{\text { S. }}{53,7}\left|\frac{\mathrm{O} .}{58,1}\right| \frac{\mathrm{N} .}{88,8} \mid \frac{\mathrm{D} .}{34,8}$

En 1963 la pluviosité fut élevée : 776,8 mm, de même qu'en 1964 (724 mm). 
1963 : Précipitations (mm).

\begin{tabular}{|c|c|c|c|c|c|c|c|c|c|c|c|}
\hline J. & F. & M. & A. & M. & J. & $\mathrm{J}$. & A. & S. & 0. & N. & D. \\
\hline 40,5 & $107, !$ & 10,4 & 31,4 & 17,3 & 19,7 & 67,2 & 23,1 & 186,7 & 87,0 & 71,6 & 114 \\
\hline 964 & & & & & & & & & & & \\
\hline 20,5 & 130 & 110,4 & 20,7 & 49,2 & 3,5 & 14,5 & 73 & 79,3 & 106,1 & 64,2 & 52,6 \\
\hline
\end{tabular}

Le climatogramme ( $f \mathrm{ig} .1$ ), établi d'après les moyennes des années 1944-1961 montre que juillet est le mois le plus sec et le plus chaud, janvier le plus froid.

En 1963, les vents se répartissaient de la façon suivante :

$\begin{array}{lrr}\text { W-SW } & 9,1 & \% \\ \text { N-NE } & 6,8 & \% \\ \text { S à E } & 19,5 & \% \\ \text { NNW } & 45,3 & \% \\ \text { calme } & 19,5 & \%\end{array}$

Toutes ces données ne correspondent que grossièrement à la réalité; d'importantes variations dans les cycles annuels peuvent survenir d'une année à l'autre. Nous pouvons actuellement distinguer de simples modifications climatiques annuelles et des modifications plus importantes de longue durée.

\section{II. - EvoluTION DU OLMAT, DU BHAN DE L'AUU ET DU SEL}

Avant l'implantation de la riziculture, vers 1944 , le bilan de l'eau et du sel en Camargue ne dépendait en grande partie que des facteurs climatiques. Les étangs saumâtres de moyenne et basse Camargue présentaient en été des niveaux très bas ou s'asséchaient, découvrant de vastes plages dénudées. Les augmentations considérables de la salinité qui en résultaient dans ces milieux étaient accentués par une évaporation estivale intense. En hiver cependant, les étangs débordaient fréquemment et inondaient les parties basses de Camargue. Cette alternance de submersions et d'assèchements avait de profondes répercussions sur les composantes floristiques et faunistiques. Nous ne possédons malheureusement que peu de données qualitatives et quantitatives de l'évolution des différents biotopes de cette époque. Celles de G. Petrt et D. Schachter ne sont souvent que trop fragmentaires pour retracer exactement cette évolution.

C'est grâce aux documents de G. Tallon, qu'il est possible de suivre les transformations des milieux aquatiques. En 1938, cet auteur trace le bilan général des eaux en Camargue avant 1937 : "Il a déjà été exposé ici à plusieurs reprises comment l'ensemble aes eaux pluviales et d'arrosage du delta, collectées au Vaccarès par un réseau de canaux, avait pris un volume de plus en plus 
considérable par suite de l'extension de la culture de la vigne; que le niveau d'hiver de la nappe générale d'eau, devenait de plus en plus élevé, submergeant les routes et même les terres sous les eaux salées. »

Cet excès d'eau provoque vers le milieu des années 30 un commencement d'évolution de la bordure des étangs, où s'installe une flore moins halophile. Les parties centrales de l'étang du Vaccarès sont occupées par de grands herbiers de Ruppia maritima. Crevettes grises, Athérines et Anguilles y pullulaient.

Or, en 1937, une période de sécheresse commence et change entièrement les niveaux d'eau et les salinités. La sécheresse sévit en été et se prolonge même pendant l'hiver. Dès 1938, G. Tallon écrit : "Cette sécheresse ne s'est pas terminée avec l'été et, après quelques faibles pluies en octobre et novembre, immédiatement bues par le sol, elle a repris en décembre. Aussi, jamais depuis le début de la réserve, n'a-t-on vu la Camargue aussi sèche en hiver. Le Vaccarès est bordé de vastes plages, toutes les baisses et même certains étangs comme le Fangassier sont entièrement sans eau, alors que pendant les premières années de la réserve, à cette époque, une même nappe d'eau recouvrait toute la basse Camargue, mettant par exemple $40 \mathrm{~cm}$ d'eau sur la route de Salin de Badon».

En 1949, G. Tallon poursuit : «Depuis 1936, les années sèches succèdent aux années sèches sans interruption en Provence... Les 'tangs salés, dits inférieurs, et le Vaccarès ont le plus souvent conservé, même en saison humide, de larges plages exondées, tandis qu'en saison sèche, ces plages occupaient la totalité ou une très grande surface de ces étangs dont l'eau restante devenait très concentrée ». Il semble que cette période de sécheresse n'ait pas été seulement la conséquence des facteurs climatiques, mais aussi celle d'une augmentation de l'évacuation d'eau par la digue à la mer et d'une diminution des irrigations pendant la guerre.

C'est en 1949, que la situation des eaux camarguaises se modifie brusquement et que "le territoire de la Réserve a repris à ce moment son aspect de submersion hivernale qui était la règle au moins pendant les 7 années antérieures à $1936 »$. [G. TALLON 1950.] Cette nouvelle submersion correspond à l'augmentation des rizières irriguées qui passent de 700 ha en 1946 à 13.500 ha en 1952 .

Dès 1951, le Vaccarès et les étangs inférieurs recevaient 200 millions de $\mathrm{m}^{3}$ d'eau supplémentaires. Ces apports d'eau de collature coïncidèrent en outre avec un retour des années pluvieuses, notamment les hivers 1951-1952 puis 1953-1954. Bientôt les plages du Vaccarès disparurent non seulement pendant l'hiver mais restèrent également inondées pendant tout l'été.

Schématiquement, aujourd'hui les milieux les plus doux se rencontrent en haute Camargue tandis que les plus fortes concentra- 
tions en sel se situent en bordure de mer et, principalement, dans les surfaces préparatoires des salines du sud-est de la Camargue. Dans la partie nord, l'influence de l'homme tend à abaisser la chlorinité, dans la partie sud, au contraire, ce même facteur tend à l'élever. Pour abaisser la salinité dans la Camargue cultivée, l'unique solution est d'irriguer ou de drainer, donc d'apporter de l'eau douce pompée dans le Rhône et de la rejeter ensuite dans les marais de moyenne Camargue où elle maintient un niveau élevé en été (Vaccarès, Fournelet...). Pour augmenter la salinité au contraire, il faut évaporer et l'opération est d'autant plus rapide que l'on prend comme point de départ une eau renfermant déjà une teneur en sel plus importante. La Camargue centrale, c'est-àdire la réserve et son voisinage, se trouve comprise entre ces deux extrêmes d'intérêts contradictoires. Elle est isolée des exploitations salinières par des digues, mais elle reçoit les eaux de collature des cultures irriguées et s'en débarrasse en partie par évaporation, rejetant le surplus à la mer. Il en résulte une diminution de la salinité, d'une part par une plus forte dilution du sel dans une masse d'eau plus importante, d'autre part, par le lessivage progressif qui se fait surtout lors des années pluvieuses.

En gardant toujours à l'esprit cet aspect schématique de la Camargue, il est possible de reconnaître 3 grandes zones : la première étant celle des régions cultivécs et irriguées, la seconde celle des marais et étangs assurant l'écoulement des eaux d'irrigation vers la mer, la troisième celle des marais et étangs recevant de l'eau de mer pour l'exploitation des salins (13.000 ha). Ces trois zones occupent la plus grande superficie du delta, mais il existe en outre quelques marais suffisamment isolés qui échappent à leur influence ou tout au moins à leur influence directe.

Actuellement, malgré les diverses transformations dues à la riziculture et à l'extension des salins, l'écologiste peut toujours trouver toute une succession de milieux échelonnés depuis l'eau douce jusqu'à l'eau sursalée.

Essayons, par quelques exemples, d'étudier les diverses vicissitudes subies par quelques milieux types et de voir quelles modifications ont entraîné la riziculture et les salins.

\section{III. - EXEMPLES DE MODIFIOATIONS SUbIES PAR LES MARAIS CAMARGUAIS}

\section{1. - LE FANGASSIER.}

Situé dans la partie sud de la Camargue, il est séparé à l'ouest par une digue de l'étang du Galabert et plus au sud du Grand Rascaillan. Nous sommes ici en présence de l'exemple type de 


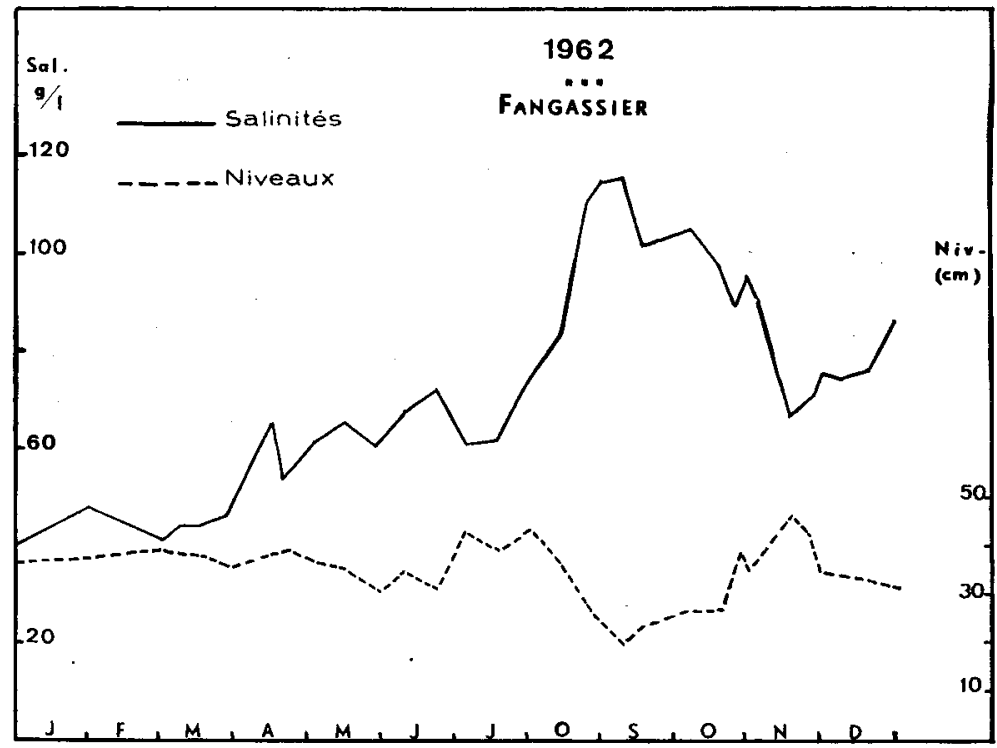

Fig. 2. Évolution des salinités et des niveaux de l'étang du Fangassier en 1962

transformation d'un étang saumâtre en un étang sursalé. La Compagnie Salıcam l'a récemment annexé dans le circuit de ses surfaces préparatoires. L’eau pompée dans la mer est acheminée dans le Galabert, passe plus au nord dans la zone des Enfores, puis revient dans le Fangassier. Avant 1961, cet étang avait une salinité voisine de celle de l'eau de mer, se concentrant en été et découvrant alors d'immenses plages limoneuses ou sablonneuses. En hiver, au contraire, les eaux de pluie et celles qui lui parvenaient librement, jusqu'au début des années 50, des étangs de la réserve, remplissaient le Fangassier et le rendaient beaucoup moins salé que la mer.

La faune des Copépodes, la mieux connue, était composéc d'Eurytemora velox, Halicyclops neglectus, Diacyclops bisetosus, Laophonte setosa, Cletocamptus retrogressus, Harpacticus littoralis espèces typiques d'eaux où la concentration en sel est voisine de celle de l'eau de mer. La flore était essentiellement formée d'herbiers de Ruppia maritima.

Depuis son annexion au circuit des salins, de profondes modifications biologiques se sont manifestées. La salinité considérablement élevée (fig. 2) pouvait atteindre en 1962, aux mois d'août et septembre, $120 \mathrm{~g} / 1$ avec un niveau d'eau assez élevé, et en moyenne de 70 à $80 \mathrm{~g} / \mathrm{l}$ en hiver. Ceci a provoqué la disparition des herbiers de Ruppia. 
A une faune d'eau saumâtre diversifiée, a succédé une faune d'eau sursalée, où l'on rencontre fréquemment l'Harpacticide Cletocamptus retrogressus, le Phyllopode Artemia salina, une ou deux espèces de Chironomides : Orthocladius sp., des larves d'Ephydra et une espèce de Coléoptère, Potamonectes cerisyi. Seules les espèces les plus euryhalines et eurythermes des milieux extrêmes formés par les salins y persistent. On assiste à une réduction très nette du nombre des invertébrés et en même temps à une pullulation des individus de chaque espèce.

\section{2. - LE VACCARES.}

Petit et Schachter [1954], ont déjà mentionné les transformations subies par le Vaccarès depuis la construction de la digue à la mer, en 1859, l'influence des cultures irriguées, et enfin la transformation importante de son régime hydrologique due à l'extension de la riziculture.

Deux points intéressants sont à retenir :

a) Avant la riziculture, le Vaccarès recevait beaucoup moins d'écoulages provenant des cultures et son régime était surtout sous l'influence des conditions climatiques. Ainsi en été le niveau baissait considérablement, découvrant d'immenses plages, et était suivi d'une augmentation de la salinité. Le 30 août 1941, Petrt et Schachter signalent au large, en face de la grande plage (cabane des pêcheurs), $82,25 \mathrm{~g} / \mathrm{l}$, le 17 septembre $1949 ; 19,65 \mathrm{~g} / \mathrm{l}$ dans la zone des Chaetomorpha.

b) La riziculture nécessitant un apport considérable d'eau en provenance du Rhône, a sérieusement augmenté les collatures. Les eaux après passage dans les rizières sont acheminées dans les canaux qui se déversent dans le Vaccarès. Le Vaccarès est devenu un immense réservoir. Du fait de ces rejets, le niveau a augmenté; Petit et Schachter [1954] citent «En 1952, malgré un été chaud et sec, l'étang a conservé à peu de chose près son niveau hivernal, et les étangs du sud ne se sont point asséchés. Depuis cette époque, le Vaccarès reste, en permanence, relativement haut $»$.

Une étude monographique complète actuellement en cours permettra de mieux saisir les changements intervenus et les répercussions sur la faune et la flore de cet étang.

Avant la grande sécheresse des années 1936 à 1949, G. TALLon signale l'abondance de Crevettes grises, d'Athérines et d'Anguilles. Nous ne disposons pas d'autres informations zoologiques, ni sur ia faune avant 1936, ni sur les répercussions des années sèches, mais il est à peu près certain qu'une partie de la faune, peu résistante au sel, a du disparaître pendant les années sèches et salées. 
D. Schachter a cependant étudié la faune vers la fin de la période de sécheresse et au début de la période d'adoucissement qui suivit. Pour les années sèches de 1941 à 1949, Petit et Schachter [1954] citent parmi les Mollusques :

Cardium (Ceratoderma) edule var. rectidens

Cardium (Ceratoderma) edule var. quadrata

Abra (Lutricularia) ovata

Rissoa labiosa var. fragilis

sur le bord de l'étang :

Paludestrina acuta

Leuconia micheli

Phytia myosotis

l'Isopode Sphaeroma hookerii, les Amphipodes Gammarus locusta et Talorchestria brito, l'Annélide Polychète Nereis diversicolor.

Le zooplancton était représenté par des populations presque pures d'un Crustacé Copépode : Eurytemora velox Lillj. «Ia faune ichtyologique qui rappelait autrefois celle de tous les étangs littoraux méditerranéen (Dorades, Turbots, Soles, Loups, Mulets, Athérines) avait déserté l'étang et l'on ne pêchait plus dans le Vaccarès que quelques rares Mulets, des Athérines et des Anguilles, sujettes parfois à une mortalité intense ».

Après 1949, avec la réduction de la salinité et l'augmentation du niveau, "les Muges ont reparu en quantité importante, ainsi que les Plies. Les Poissons chats (Amiurus nebulosus Lesueur) sont plus nombreux que jamais. Des espèces jusqu'alors cantonnées dans les roubines, Abramis brama L., Tinca tinca L., s'y rencontrent aujourd'hui, ainsi que les Carpes (Cyprinus carpio L.), les Carassins (Cyprinus kollari), les Brochets et les Perches-soleil (Eupomotis gibosus L.)... Corrélativement, les populations planctoniques d'Eurytemora velox se trouvaient progressivement remplacées par des populations denses de Calanipeda aquae dulcis ».

Les salinités qui se situaient en 1947 aux environs de $24 \mathrm{~g} / \mathrm{l}$ en mai, $35 \mathrm{~g} / \mathrm{I}$ en juin à la pointe de Mornès (partie sud du Vaccarès), sont tombées en 1953 aux environs de $10 \mathrm{~g} / \mathrm{l}$ en mai et $9 \mathrm{~g} / \mathrm{l}$ en juin à cette même station.

Les modifications subies par la flore et notamment l'extension considérable de la roselière (à la Capelic̀re entre autre) sont traitées en détail par G. TalloN. Cet auteur constate en même temps la disparition des plages en été, et de la végétation terrestre qui les recouvre. Cette évolution s'est maintenue jusqu'à nos jours.

Depuis 1954, nous suivons cette évolution. Ces données seront reprises en détail dans une publication ultérieure. Toutefois, nous pouvons résumer dès à présent les caractéristiques essentielles actuelles du Vaccarès. 
Salinités.

On peut dire que la riziculture après une phase expansive s'est stabilisée depuis 1962. Aussi plusieurs stations de refoulement des eaux de collature au Rhône ont été construites pour dégager un peu le Vaccarès. Ceci a amené une stabilité relative de la salinité. Au cours de nombreux relevés, on constate qu'actuellement la salinité varie de 6 à $10 \mathrm{~g} / \mathrm{l}$ avec une moyenne de $8 \mathrm{~g} / \mathrm{l}$ au centre de l'étang (août 1962 et 1963). Ceci correspond en fait à des salinités élevées malgré l'influence de 20 années de riziculture.

\section{Intérêt faunistique.}

A l'exception des roselières, parties les plus périphériques situées à proximité de l'embouchure des grands canaux, peuplées d'une faune à tendance dulçaquicole ou faiblement oligosaumâtre, on note que la majorité du peuplement est typique des eaux saumâtres-mésopoikilohalines (minimum oligosaumâtre), soit qu'il se soit maintenu par suite de l'euryhalinité des espèces, ou qu'ill soit récemment apparu grâce à des conditions hydrologiques plus stables.

Les populations planctoniques d'Eurytemora velox, espèce très euryhaline, ont disparu au profit d'une autre espèce de Copépode : Calanipeda aquae dulcis. Parmi les espèces du macrobenthos :

Gammarus locusta cf. camarguensis

Sphaeroma hookeri

Nereis diversicolor

Idotea viridis

Crangon crangon

s'y rencontrent en abondance.

D'importants changements ont eu lieu dans la faune malacologique. Les Rissoa ont disparu; ne persistent que Abra ovata et Cardium glaucum. On assiste toujours à des mortalités importantes des Cardium qui viennent s'accumuler sous l'action des vents et du brassage des eaux, sur les berges. L'Hydrobiidae Hydrobia acuta a été progressivement remplacé par Hydrobia ventrosa, espèce plus euryhaline.

\section{Intérêt floristique.}

Nous emprunterons à G. TALLoN les plus importantes observations sur les données floristiques. En 1938 il notait : «Il y avait un commencement d'évolution dans la bordure végétale des étangs, qui tend bers l'installation d'une flore moins halophile, mais c'est dans le milieu même des eaux du Vaccarès que les changements ont été les plus profonds. Ils sont même tellement importants, que lon peut se demander si d'autres causes ne sont point intervenues. Les grands herbiers de Ruppia maritima, plantes phanérogames annuelles, submergées, un peu analogues aux Zostères, qui recou- 


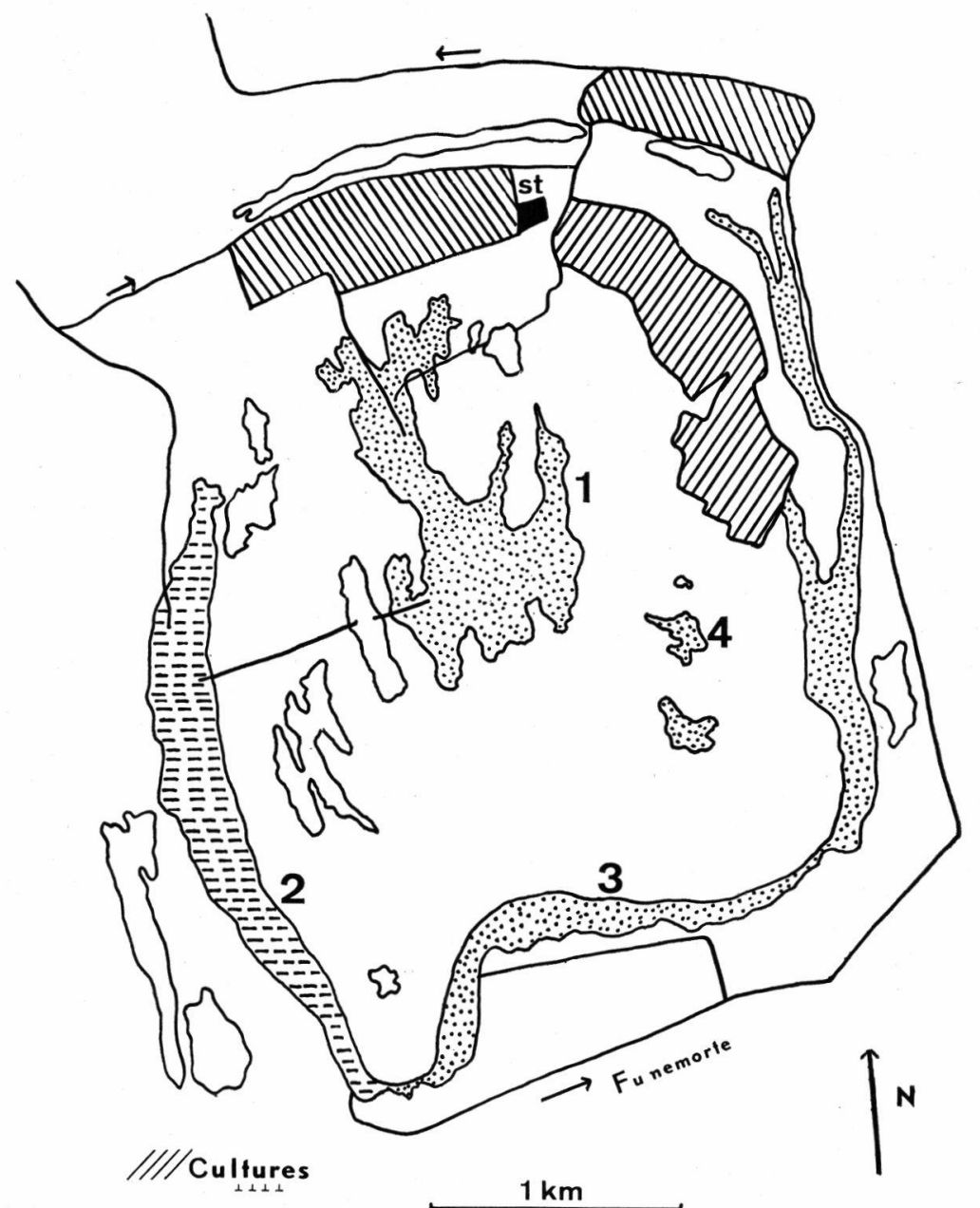

Fig. 4. Stations régulièrement prospectées dans les marais de la Tour du Valat : 1) Saint-Seren; 2) Baisse-Salée; 3) Relongues; 4) Moyenne Cerisière.

vraient toute la partie peu profonde de l'étang, ont été réduits à peu de chose. Par contre est apparu, en quantité chaque jour plus considérable, une algue flottante qui se présente sous la forme de gros crins verts; ce sont des Chaetomorpha». Ces observations sont valables pour la période de 1936-1949.

A partir de 1949, le retour d'années humides auquel s'ajoute l'influence croissante des apports d'eau douce provenant des cultures irriguées, a favorisé une «dessalure »générale et provoqué l'extension dans toute la partie nord de l'étang d'une zone d'herbiers de Potamogeton pectinatus. Les immenses roselières de 
Phragmites communis n'ont cessé de s'accroître dans les zones les plus douces recevant des écoulages.

Dans la partie sud de l'étang un peu plus salée $(10 \mathrm{~g} / \mathrm{l}$ environ en août 1963 et 1964), ont persisté les herbiers de Ruppia maritima. Une flore alguale typique d'eau saumâtre a envahi l'étang :

Chaetomorpha linum

Polysiphonia flocculosa

Monostroma oxycoccum

Enteromorpha intestinalis

A noter aussi parmi les herbiers de Ruppia et Potamogeton, d'importantes zones de Chara sp.

D'après nos observations régulières depuis 1962 , la situation actuelle paraît se stabiliser, le Vaccarès restant un étang saumâtre aux conditions plus stables qu'antérieurement, mais du plus haut intérêt pour l'écologiste.

\section{3. - LE SAINT-SEREN.}

Ce type de marais se rencontre fréquemment en moyenne Camargue où il forme un des milieux les plus caractéristiques.

Non entièrement clos, il reçoit de l'eau douce provenant d'apports d'écoulages de rizières; de plus, il est peu profond (maximum 80 à $100 \mathrm{~cm}$ en période des hautes eaux) et pâturé par les taureaux. Il peut s'assécher en été ou être maintenu en eau soit par les apports artificiels d'eau douce, soit par de fortes pluviosités estivales.

Les premiers relevés faunistiques partiels datent de 1953. A partir de 1955, nous possédons des renseignements quantitatifs et qualitatifs réguliers. Avec l'extension de la riziculture, ce marais s'asséchait l'été et devait ressembler aux cerisières actuelles, marais temporaires oligosaumâtres-oligopoikilohalins, soumis uniquement aux conditions climatiques. Il est probable que le cycle hivernal, avant 1953, était compris entre 1 ou $2 \mathrm{~g} / \mathrm{l} \mathrm{NaCl}$ et, qu'à l'assèchement, cette salinité restait encore assez faible, ne dépassant pas $5 \mathrm{~g} / \mathrm{l}$. Les précipitations abondantes, créant des inondations pendant les hivers 1951-1952 et 1953-1954, apportèrent de l'eau plus saléc venant des étangs saumâtres. Ainsi la salinité atteignait $10,3 \mathrm{~g} / \mathrm{l}$ le 22-9-1955 dans les zones les moins profondes. De 1953 à 1960, la flore était formée d'herbiers de Scirpus maritimus et de Chara, avec, dans les parties les plus profondes, des Potamogeton pectinatus scoparius et Myriophyllum spicatum et, sur le bord, des Ranunculus baudotii.

L'un d'entre nous a relevé, en 1956, quelques vestiges d'une végétation assez différente qui devait antérieurement coloniser ce 


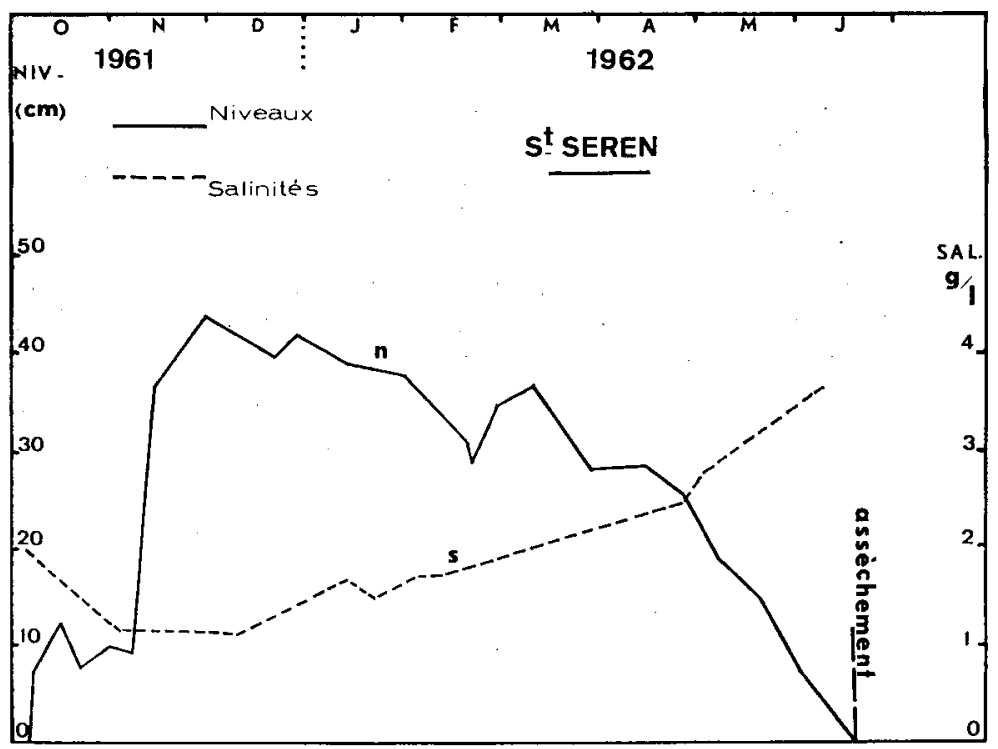

Fig. 5. Marais du Saint-Seren, Evolution de la salinité et des niveaux pendant le cycle 1961-1962.

milieu. La partie centrale de la cuvette la plus profonde devait être occupée par des Scirpus maritimus avec en bordure un Junceto triglochinetum maritimi et dans les parties hautes : Heleocharis palustris et Juncus gerardi. Ce peuplement est identique à celui qui actuellement colonise les Cerisières.

\section{Salinité.}

Nous ne reprendrons pas en détail la description des cycles [P. Aguesse 1958, F. Marazanof 1963].

De l'examen de la figure 5 et 6 , il est facile de tirer des conclusions générales.

Alors que la salinité varie actuellement de 1,1 à $2 \mathrm{~g} / \mathrm{l}$ pendant la période hivernale (niveau des plus hautes eaux), elle ne dépasse pas $5 \mathrm{~g} / \mathrm{l}$ en période estivale.

Depuis 1962, ce marais ne s'est pas complètement asséché, ceci malgré une baisse importante des niveaux en août et septembre 1963 et 1964, où la salinité n'a pas dépassé $5 \mathrm{~g} / \mathrm{l}$.

Par contre, en 1955, 1956 et 1957, pendant l'été, la salinité pouvait atteindre et dépasser $7 \mathrm{~g} / \mathrm{l}$; le 16 juillet $1962, \mathrm{P}$. Heurteaux a relevé avant l'assèchement $16,8 \mathrm{~g} / 1$.

L'apport de plus en plus fréquent d'eaux de collatures a, depuis cette date, favorisé l'abaissement des teneurs en sel. Les eaux oligosaumâtres-mésopoikilohalines de ce marais (moyenne et mini- 
mum de salinités compris entre 0,5 et $5 \mathrm{~g} / \mathrm{l}$, maximum entre 5 et $16 \mathrm{~g} / \mathrm{l}$ ), tendent progressivement depuis 1962 vers la catégorie inféreure d'eaux oligosaumâtres-oligopoikilohalines.

\section{Conséquences faunistiques.}

A une faune d'eaux oligosaumâtres-mésopoikilohalines formée surtout d'Harpacticides (Nitocra lacustris, Onychocamptus mohammed), de Centropagides (Calanipeda aquae dulcis et Arctodiaptomus wierzejskii), de Cladocères comme Daphnia magna, Chydorus sphaericus, Alona rectangula, Simocephalus vetulus succède un peuplement dulçaquicole typique formé essentiellement à l'heure actuelle de Cyclopides :

Megacyclops viridis

Diacyclops bicuspidatus

Acanthocyclops robustus

et de Cladocères identiques aux précédents mais auxquels viennent s'ajouter :

Daphnia longispina

Scapholeberis mucronata

Acroperus harpae

Daphnia pulex

Ces formes dulçaquicoles tendent à éliminer les espèces les plus oligohalines. La faune de ce marais est une faune d'apport, qui se retrouve soit dans les canaux d'eau douce peu profonds, soit dans les marais oligosaumâtres-oligopoikilohalins permanents envahis par une abondante végétation émergente de Typha et de Phragmites.

La faune malacologique actuelle du Saint-Seren est une faune provenant des roubines avoisinantes et qui, du fait de l'adoucissement général du milieu, s'y développe abondamment; tel est le cas de :

Physa acuta

Lymnaea palustris

L. peregra

L. auricularia

Ancylus fluviatilis

Bithynia tentaculata

On pourrait multiplier les exemples : les Hétéroptères, Odonates, Coléoptères... y sont bien représentés.

Remarque. - Si l'on considère les fig. 5,6 et 7 , il est intéressant de noter l'influence fondamentale jouée par la permanence ou l'assèchement des eaux entre deux cycles biologiques successifs. Ce facteur conditionne l'apparition ou la disparition de certaines espèces planctoniques; ainsi, si le marais s'assèche en été (voir 


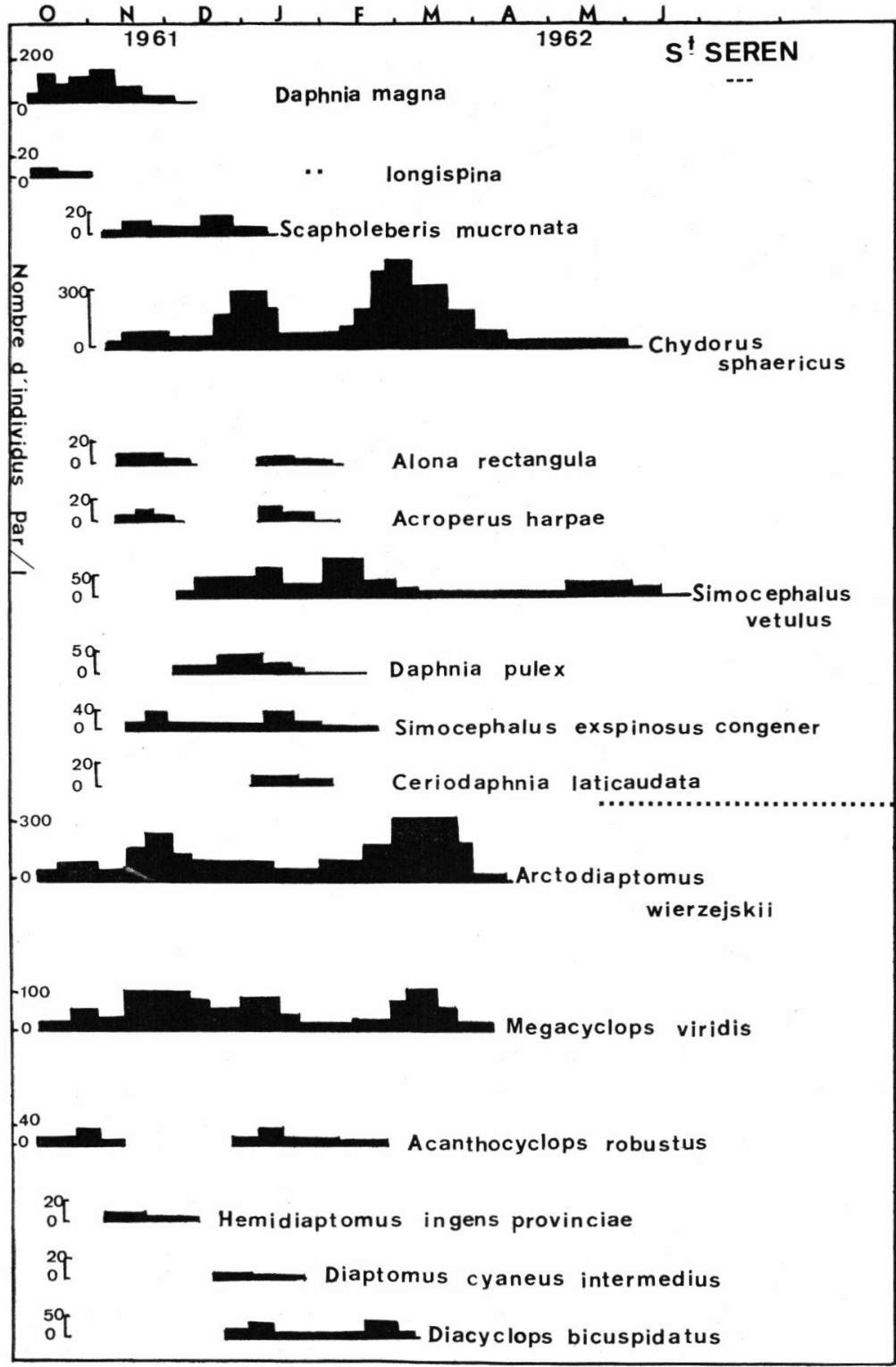

Fig. 7. Variations quantitatives des populations planctoniques de Copépodes et Cladocères au Saint-Seren pendant le cycle 1961-1962 (valeurs exprimées en nombre d'individus par litre d'eau).

cycle 1961-1962), il apparaît toujours, dans le cycle qui commence avec les pluies d'automne, le Copépode Arctodiaptomus wierzejskii et le Cladocère Daphnia magna. 
Si le marais ne s'assèche pas entre deux cycles successifs, ces ùeux espèces, qui normalement pullulent, ne réapparaissent pas et sont remplacées par Calanipeda aquae dulcis, espèce très euryhaline que l'on retrouve dans le Vaccarès, et les Harpacticides

Onychocamptus mohammed

Nitocra lacustris

Quantitativement, la faune planctonique dans ce dernier cas, est très pauvre.

Le peuplement actuel du Saint-Seren est un peuplement d'eau douce banal analogue par exemple à celui du marais des l'Esquineau de la Tour du Valat, marais d'eau douce faiblement oligosaumâtre. Le 20-3-1962, $\mathrm{NaCl}=0,75 \mathrm{~g} / \mathrm{l}$, l'un d'entre nous a relevé dans ce marais les Copépodes :

Megacyclops viridis

Acanthocyclops robustus

Onychocomptus mohammed

Diacyclops bicuspidatus

et les Cladocères :

Daphnia longispina

Scapholeberis mucronata

Alona rectangula

Acroperus harpae

Ceriodaphnia laticaudata

Chydorus sphaericus

\section{Conséquences floristiques.}

Les conséquences de l'adoucissement progressif de ce milieu ont été très nettes sur les composantes floristiques.

La partie nord, zone des écoulages, est de plus en plus envahie par les Phragmites communis et les Typha angustifolia. Les Scirpus maritimus ne formant, en 1955, qu'une ceinture périphérique, n'ont cessé d'envahir le marais et de coloniser les parties les plus profondes. Récemment sont apparus, d'après A. TAmisier (observations inédites), des herbiers de Potamogeton pusillus. Heleocharis palustris est fréquent dans la partie nord.

Depuis que le marais ne s'assèche plus, les herbiers de Potamogeton pectinatus et de Myriophyllum spicatum sont devenus plus denses. Les Chara sp. et les Zanichelia sp. se maintiennent, de même que les Ranunculus baudotii. Des relevés réguliers de flore nous font défaut, mais, incontestablement, la productivité primaire a considérablement augmenté depuis $\mathbf{1 9 6 2}$.

C'est une évolution en fait très rapide; le marais "se ferme", envahi par une abondante végétation aquatique émergente de $\boldsymbol{S c i r}$ pus maritimus, réduisant de plus en plus les parties d'eau libre. A noter aussi l'installation récente de touffes de Scirpus littoralis et de Scirpus lacustris (communication de A. Tamisier). 


\section{4. - BAISSE-SALEE ET RELONGUES.}

\section{Baisse Salée de la Tour du Valat.}

Marais oligosaumâtre-mésopoikilohalin peu profond, à fond limoneux. Les premières observations sont mentionnées par D. Schachter [1950] puis par P. Aguesse et L. Bigot [1960] et F. Marazanof [1963].

Ce marais a subi des transformations dues à la riziculture. On assiste également dans ce milieu au remplacement très net des espèces à tendance halophile par des espèces faiblement oligohalines et dulçaquicoles. Au point de vue floristique, l'enhavissement progressif de la partie nord par les Phragmites et les Typha angustifolia est très net. Si l'avancée de Scirpus maritimus est moins spectaculaire qu'au Saint-Seren, les Scirpus littoralis et Scirpus lacustris forment des touffes de plus en plus importantes. Les herbiers de Potamogeton pectinatus, Myriophyllum spicatum, Chara sp. et de Ranunculus baudotii y sont de plus en plus denses.

Afin d'éviter cette tendance à l'adoucissement, de l'eau plus salée en provenance du Fournelet a été introduite en 1964 (salinité de $20 \mathrm{~g} / 1$ au mois d'août 1964).

Il est encore trop tôt pour tirer les conséquences de cet apport de sel car l'installation d'une évolution régressive et l'apparition nouvelle d'une biocénose, demandent parfois plusieurs années. Il est probahle qu'une faune et une flore d'eau saumâtre s'y réinstalleront.

\section{Les Relongues.}

Ce marais du même type que les deux précédents a aussi subi un net adoucissement. Alors qu'en 1962, on relevait en période d'assèchement des salinités de $212 \mathrm{~g} / \mathrm{l}$ le 8 juillet, avec des traces de dépôts où pullulaient des Gammarus locusta [F. Marazanof 1963], ces conditions extrêmes ont disparu pendant les années 1963-1964. Le creusement d'un canal, en 1962, destiné à alimenter ce marais en eau, n'a fait qu'accentuer l'adoucissement et entraîné le remplacement d'un peuplement euryhalin par un peuplement d'eau douce.

En 1963, la salinité maximum, n'a pas dépassé $5,3 \mathrm{~g} / \mathrm{l}$ et s'est maintenue pendant la période hivernale de 1963-1964 au-dessous de $2 \mathrm{~g} / \mathrm{l}$. L'envahissement de ce marais par les Scirpus maritimus et les Phragmites est aussi spectaculaire qu'au Saint-Seren. 


\section{5. - LES CERISIERES.}

II existe en Camargue un certain nombre de mares, peu profondes, à l'écart de toute transformation humaine et d'apports extérieurs provenant de cultures irriguées, formant un des milieux les plus stables uniquement influencé par les facteurs climatiques.

La faune et la flore de ces marais couramment appelés Cerisières sont bien connues : P. Aguesse [1956, 1957], P. Aguesse et M. Nourisson [1961], F. Marazanof [1963].

La salinité de ces mares ne dépasse jamais au cours d'un cycle annuel $3 \mathrm{~g} / \mathrm{l}$. Généralement mises en eau avec les pluies d'automne, elles maintiennent un niveau d'eau élevé pendant tout l'hiver et ne s'assèchent qu'à la fin du printemps. Les variations dans le cycle de la faune sont ici annuelles et intimement liées aux conditions climatiques, il suffit de retrouver ces mêmes conditions pour voir réapparaître la même faune.

A la phase aquatique succède en été une phase terrestre où s'installe une flore composée de Polygonon maritimum, Lythrum hyssopifolium, Typha australis et Heleocharis palustris.

De nombreuses espèces d'invertébrés aquatiques paraissent trouver uniquement dans ces biotopes un milieu propice à leur développement. Seuls persistent les Crustacés possédant des œufis de résistance. C'est le cas des Diaptomides :

Mixodiaptomus kupelwieseri

Diaptomus cyaneus intermedius

des Phyllopodes :

Tanymastix lacunae

Chirocephalus stognalis

Branchipus stagnalis

Imnadia yeyetta

Triops cancriformis

Il est possible que l'on soit ici en présence de milieux « relictes", qui se sont maintenus à l'écart de toute transformation, abritant une faune d'eau temporaire, généralement riche en espèces. On peut imaginer que le Saint-Seren, avant l'influence des écoulages, avait l'aspect de ces mares et était peuplé d'une faune à peu près identique. Cette hypothèse est d'autant plus plausible qu'en 1962 le Saint-Seren s'est asséché et a retrouvé un caractère temporaire. A la remise en eau de l'automne, des populations de Diaptomus cyaneus intermedius et de Hemidiaptomus ingens provinciae (cf. fig. 7), espèces uniquement inféodées aux Cerisières, s'y sont développées.

L'évolution de la flore dans ces mares temporaires est beaucoup plus lente que celle du Saint-Seren et est caractérisée par une faible colonisation du Scirpetum maritimi et de la Phragmitae. 


\section{IV. - LES GRANDS TRAITS DE L'ÊVOLUTION DES MIUEUX}

Ces quelques exemples de transformations subies par les marais camarguais sont du plus haut intérêt pour comprendre les changements successifs faunistiques et floristiques. Nous sommes en présence de deux évolutions différentes et opposées, mais toutes deux dues à l'homme.

\section{1. - MODIFICATIONS DUES AUX SALINES.}

L'implantation des bassins d'évaporation dans la partie sud-est de la Camargue, s'est faite au détriment de zones douces ou saumâtres. Aux étangs naturels avec leurs fluctuations importantes succèdent des étendues artificielles, où les niveaux et les salinités dans les différents plans d'eau doivent être maintenus constants de inars à septembre. Dans certains étangs, des eaux saturées sont même maintenues pendant l'hiver.

L'évolution subie par le Fangassier est typique de cette transformation. D'une faune d'eau saumâtre et polysaumâtre, on passe progressivement à une faune et une flore d'eau salée, voire sursalée.

Les espèces de Copépodes comme

Eurytemora velox

Halicyclops neglectus

Laophonte setosa

Haipacticus littoralis

sont remplacées par une seule espèce d'Harpacticide, Cletocamptus retrogressus.

Les Gammaridae, les poissons comme les Muges (Mugil cephalus, capito et auratus), les Loups (Morone labrax), les Athérines (Atherina mochon), vivant jusqu'à des salinités de $65 \mathrm{~g} / \mathrm{l}$, disparaissent complètement. Les Mollusques

Hydrobia acuta

Hydrobia ventrosa

Cardium glaucum

quantitativement bien représentés dans les eaux saumâtres, disparalssent également.

De 5 espèces de Turbellariés, on passe à une seule espèce du genre Macrostomum (communication de Ulfert GraEfe). Ne persistent qu'une espèce de Chironomide : Orthocladius sp., des larves d'un Diptère du genre Ephydra, une espèce de Phyllopode, Artemia salina, et une espèce de Coléoptère, Potamonectes cerisyi.

On assiste à une raréfaction de la faune, et une persistance de quelques espèces qui par leur grande euryhalinité et eurythermie, arrivent à se maintenir et souvent à pulluler. Quelques oiseaux comme les Flamants, les Avocettes, les Mouettes rieuses et les Larolimicoles profitent de ces quantités importantes de nourriture. 


\section{2. - MODIFICATIONS DUES AUX RIZIERES.}

Nous décomposerons les grands traits en 2 stades d'adoucissement progressif.

\section{Premier stade.}

La succession faunistique et floristique observée dans le Vaccarès est typique. Aux brusques variations de salinités et de niveaux a succédé un plan d'eau beaucoup plus stable. En 1963 par exemple, le niveau du Vaccarès variait entre la cote -27 et +28 par rapport au $\mathrm{O} N \mathrm{NGF}$., ce qui représente une faible amplitude. Après une forte diminution de la salinité, par augmentation du volume d'eau et par lessivage, les conditions sont devenues plus stables depuis quelques années. La salinité n'a guère varié ces dernières années et se maintient aux environs de 7 à $10 \mathrm{~g} / \mathrm{l}$ en été. Ceci doit être en partie du au repompage d'une fraction des eaux de collatures vers le Rhône.

Il n'est guère probable que le Vaccarès s'adoucisse beaucoup plus. C'est d'autant plus invraisemblable qu'une nappe salée, peu profonde, a été décelée dans les sédiments en 1964 sous le Vaccarès (prospection sédimentologique, faunistique et floristique effectuée par P. Heurteaux et F. Marazanof).

Faune. - Dans le plancton des populations pures du Copépode Eurytemora velox ont été remplacées par une autre population pure de Calanipeda aquae dulcis.

Aux poissons marins, ont succédé des poissons d'eau douce ave: un nombre plus important d'espèces.

Parmi la faune malacologique, Cardium glaucum, Hydrobia acuta et Abra ovata se sont maintenus, mais d'abondantes populations d'Hydrobia ventrosa ont colonisé le Vaccarès. Les Rissou, - espèce signalée par Paulus [1949], Petit et Schachter [1954], -. n'ont pas été retrouvés. Ne persistent que des formes d'eaux saumâtres typiques, les unes d'origine marine, les autres dulçaquicoles mais toutes euryhalines et eurythermes. Les espèces benthiques qui $\mathrm{y}$ vivent, comme Sphaeroma hookeri, Idotea viridis, Gammurus locusta $c f$. camarguensis, Crangon crangon et les espèces planctoniques sont d'autant plus résistantes que le Vaccarès subit pendant l'année l'influence des vents dominants. Le mistral en particulier provoque, par suite de la faible profondeur de l'étang, un brassage considérable des eaux. Le Vaccarès demeure malgré tout un étang où la faune est réduite et où la productivité secondaire reste faible.

Au point de vue de la productivité primaire, on assiste à un enrichissement, depuis l'influence de la riziculture. Les herbiers de Potamogeton pectinatus, de plus en plus denses dans toute la partie nord, tendent à coloniser les parties les moins profondes vers le sud et à remplacer les Ruppia maritima. 
D’autre part, l'adoucissement paraît avoir provoqué le développement important d'algues macroscopiques comme

Chaetomorpha linum

Monostroma oxycoccum

Enteromorpha intestinalis

Polysiphonia flocculosa

(déterminations de $M$. et $\mathbf{M}^{\mathrm{me}}$ Huvé). Nous ignorons ce qu'il en est pour le phytoplancton.

\section{Deuxième stade.}

Ce deuxième stade évolutif nous est donné par la transformation de marais tels que le Saint-Seren, la Baisse Salée et les Relongues de la Tour du Valat. Par un lessivage plus intense qu'au Vaccarès, la salinitié $y$ est tombée bien davantage et ne dépasse plus les $5 \mathrm{~g} / \mathrm{l}$ même à la fin de l'été lorsque le sel est considérablement concentré.

Calanipeda aquae dulcis, quantitativement peu représenté, est remplacé par le Diaptomide Arctodiaptomus wierzejskii, les Cyclopides

Megacyclops viridis

Acanthocyclops robustus

Diacyclops bicuspidatus.

Aux Cladocères

Daphnia magna
Chydorus sphaericus
Simocephalus vetulus

fréquents dans les Relongues et la Baisse Salée, pendant le cycle 1961-1962, viennent s'ajouter dans le Saint-Seren, milieu plus doux, d'autres espèces comme

Daphnia longispina

Scapholeberis mucronata

Acroperus harpae.

Daphnia pulex

Simocephalus expinosus

Ceriodaphnia laticaudata

Des groupes tels que les Odonates, Hétéroptères, Coléoptères, Diptères sont bien représentés alors qu'ils sont très rares dans les eaux saumâtres et polysaumâtres.

La faune malacologique est une faune d'eau douce capable de résister à de faibles variations de salinité.

Dans les milieux les plus transformés par les rizières, la faune est identique à celle des canaux et des marais à roselières.

A mesure que la salinité décroît, le nombre d'espèces invertébrées augmente, mais les espèces rares sont remplacées par des espèces banales. La faune perd son originalité. 
Au point de vue de la flore, l'adoucissement provoque l'apparition de plus en plus fréquente d'espèces d'eau douce et l'accroissement des herbiers. Les Scirpus maritimus, formant la ceinture interne des marais, colonisent les parties les plus profondes et à une vaste surface d'ean libre succède une abondante végétation émergente. Le marais «se ferme». D'autre part, les herbiers de Potamogeton pectinatus, de Myriophyllum spicatum et de Chara sont plus denses dans ce type de marais. Il semblerait que l'on assiste depuis plusieurs années à une extension de plus en plus importante du Potamogeton pectinatus. Signalons que les graines de cette espèce et des Scirpus, sont intéressantes pour la nourriture de certaines espèces d'Anatidés.

Dans les zones proches des écoulements d'eaux usées, les Phragmites et les Typha s'étendent aussi de façon spectaculaire.

- Les modifications apportées par les salines amènent un appauvrissement très considérable du nombre des espèces d'invertébrés mais ceux-ci peuvent pulluler et atteindre des biomasses considérables en certaines saisons.

- Les modifications apportées par les rizières conduisent d'abord à un appauvrissement de la faune tant par le nombre des espèces que par la biomasse totale (premier stade); mais peu à peu, si la « dessalure » s'accentue et se maintient, une faune d'eau douce riche en espèces s'installe (deuxième stade). Les individus de certaines espèces sont nombreux mais nous avons alors un peuplement tout à fait banal, que l'on peut trouver dans la plupart des régions marécageuses françaises (Vendée, Sologne, les Dombes par ex.).

Au lieu de 4 ou 5 espèces qui pullulent comme dans les salines, le nomòre d'espèces est considérable avec quelques dominantes, mais le peuplement est moins original et typique des eaux douces.

\section{V. - CONCLUSION}

Ces constatations ne sont basées que sur l'observation de quelques groupes zoologiques et il serait intéressant d'étudier dans quelle mesure elles s'appliquent à d'autres groupes invertébrés et vertébrés.

La riziculture et l'industrie salinière conduisent à une homogénéisation des milieux et enlèvent de son originalité au peuplement des eaux camarguaises. Sur un autre plan, la création de ces milieux plutôt homoihalins a évidemment ajouté des éléments nouveaux pour l'écologiste. Mais il est toujours possible de rencontrer en Camargue, sur une surface évidemment réduite, tous les milieux 
intermédiaires, de l'eau douce jusqu'à l'eau sursalée, toutes les formes, des plus sténothermes et sténohalines, aux plus eurythermes et euryhalines. C'est cette gamme complète qui fait l'intérêt principal de la Camargue pour l'écologiste. L'augmentation des biotopes d'eau douce paraît d'autre part justifier l'apparition d'espèces nouvelles de plus en plus fréquentes dans le delta.

Par rapport aux milieux d'eau douce ou saumâtre qu'ils évincent, les salin's créent une vaste zone assez homogène où, à salinité égale, nous retrouvons toujours une faune identique. Ces zones ne sont pas dépourvues d'intérêt. Si elles permettent d'établir les limites de résistance aux salinités pour certaines espèces, elles contribuent aussi par l'originalité de leur faune à créer un milieu biologique intéressant. Comme le souligne L. Hofrmans [1962], " les surfaces d'évaporation sont un milieu de nidification au moins équivalent aux étangs. Au cours de l'été, à la suite d'une forte évaporation, les étangs baissent leur niveau et concentrent leur salinité, tandis que les salins sont maintenus artificiellement constants. La baisse des premiers conduit à leur scission et provoque dans un étang après l'autre une concentration de la faune invertébrée et des poissons qui attire beaucoup de Laridés, de Limicoles, d'Ardéidés, d'Anatidés et de Flamants. Dans les étés secs, ceci peut cependant mener jusqu'à un assèchement presque général et les ressources des surfaces de préévaporation, renouvelées régulièrement par un appoint de la mer, fournissent alors une compensution pour les Echassiers et les Palmipèdes ».

Dans les milieux encore plus salés, titrant de $70 \mathrm{~g} / 1$ à $200 \mathrm{~g} / \mathrm{l}$, les Flamants, les Mouettes rieuses et les Larolimicoles, peuvent profiter à certains moments d'une faune quantitativement plus riche que dans n'importe quel autre milieu camarguais.

Si la riziculture a créé pour le biologiste un nouveau milieu dulçaquicole riche en espèces, son développement jusqu'en 1962 (19000 ha) s'est fait au détriment des milieux naturels, de pâturages halophiles et parfois de marais préalablement drainés et asséchés. Ainsi, d'importants marais à roselières comme la Grand Mar et le marais de Saliers ont sérieusement été transformés au profit des rizières. Actuellement, quelques vestiges de ces milieux qui caractérisaient le nord de la Camargue, persistent encore dans les parties les plus basses.

L'étude approfondie et quantitative de nos observations devrait nous permettre de prévoir dans une certaine mesure l'avenir de la faune et de la flore camarguaise et d'apporter éventuellement remède à des évolutions défavorables. Si l'on s'appuie sur nos observations de 1953 à 1957 , la pluviosité resta très importante même en été (1956 surtout), ce qui contribua beaucoup à augmenter les volumes d'eau douce reçus par les étangs et marais de Camargue. 
Il semble bien qu'en 1956 , le dessalement et le maintien du niveau de l'eau en été aient atteint leur maximum. Le repompage au Rhône, depuis cette date, d'une partie de plus en plus importante des eaux de collatures et la diminution des pluies amenèrent ensuite une diminution des niveaux d'eau. En 1958, nombreux furent en Camargue les marais et étangs qui présentèrent des plages découvertes assez importantes ou même ceux qui furent entièrement à sec. Les salinités augmentèrent notablement, atteignant en été plus de $40 \mathrm{~g} / 1$ au Fournelet, concentration inconnue dans cet étang depuis de nombreuses années. Le Vaccarès lui-même avait retrouvé ses plages. C'est en effet la première fois depuis 1954, que nous y avons trouvé une flore terrestre de pionniers que G. Tallon a antérieurement décrite et qui est caractéristique des plages temporaires et étangs camarguais. (Kochia hirsuta, Salsola kali, Sueda maritima, Salicornia herbacea, etc...). C'est encore cette flore que nous avons rencontrée sur les plages du Fournelet et de nombreux marais de la Tour du Valat pendant l'été 1958. Les mêmes observations furent faites en 1962 (année sèche : 417,4 mm). La cote la plus basse du Vaccarès atteignait en septembre -47 par rapport au 0 NGF. Au cours de ce même mois, la salinité du Fournelet passa à $72 \mathrm{~g} / 1$. A une faune d'eau saumâtre, à peu près analogue à celle du Vaccarès succéda une faune typique d'eaux polysaumâtres et salées.

Il serait possible, - sans nuire aux installations des salins ni à la riziculture, - de retrouver dans le Vaccarès, le Fournelet et tous les systèmes des étangs inférieurs, la physionomie d'antan, avec des salinités plus élevées. Il suffirait, dans le cadre de l'aménagement général des plans d'eau camarguais, d'abaisser le niveau du Vaccarès; en été apparaitraient à nouveau des conditions rappelant celles qui dominaient avant l'exploitation rizicole.

Il est certain que cet aménagement demande des investissements considérables, mais non insurmontables (augmentation du rejet des eaux douces par pompage direct dans le Rhône, drainage amélioré de certaines zones cultivées en facilitant l'écoulement des eaux usées vers le Rhône).

Il serait urgent de conserver et d'aménager rationnellement les 135000 ha de cette Réserve naturelle, berceau d'une nature primitive des plus riches et unique en Europe occidentale.

Nous tenons à remercier le $\mathrm{D}^{\mathrm{r}}$ Luc. Hoffmann pour les précieux renseignements qu'il nous a fourni et les critiques apportées à ce manuscrit 


\section{RÉSUMÉ}

Les auteurs, s'appuyant sur les observations faites par de nombreux chercheurs depuis 1935 et sur leurs propres travaux commencés en 1953, décrivent de façon succinte les modifications de quelques étangs ou marais camarguais pris comme exemples. Selon la localisation du milieu aquatique, les variations sont dues à l'influence de l'homme (salines et rizières) ou à celle du climat (précipitations atmosphériques). La flore et la faune aquatique subissent des changements qui se reproduisent identiques à eux-mêmes lorsque les conditions de milieu se modifient de la même façon. Ainsi, pour tel cycle annuel de salinité et pour une eau temporaire, la flore et la faune seront composés de telles espèces. Pour tel autre cycle de salinité et pour une eau permanente, les composantes faunistiques et floristiques seront différentes, mais, si les facteurs du milieu sont à nouveau ce qu'ils étaient lors du premier cycle, on retrouve les plantes et les animaux qui le caractérisaient.

L'augmentation de la concentration en sel provoque une diminution du nombre des espèces, mais il y a une prolifération du nombre des individus. La diminution de la concentration, jointe à la permanence du milieu, entraîne une augmentation du nombre des espèces, mais avec relativement peu d'individus. La productivité primaire est cependant considérablement accrue.

Les espèces qui donnent à la Camargue son originalité ne se trouvent qu'en eaux saumâtres. Dans les caux adoucies, on ne rencontre que des espèces banales.

Toutes les modifications observées apparaissent aux auteurs comme réversibles : il serait néanmoins urgent de préparer un aménagement du delta sur des bases scientifiques, qui lui conserverait son originalité sans nuire aux intérêts des riziculteurs ou des industries salinières.

\section{Changes in water habitats in the Camargue over the past 30 YEARS.}

The authors have made use of the observations of numerous researchers since 1935 and the results of their own works begun in 1935 to describe succintly the changes in various sample ctangs and marshes of the Camargue. According to the location of the waters, variations found are due to the influence of man (salt industry + rice cultivation) or climate (atmospheric precipitation). Identical changes in aquatic flora and fauna occur each time the same environmental changes occur. Thus for a given cycle of salinity in a temporary body of water, the flora and fauna will be composed of certain given species. For another given salinity cycle in a permanent body of water, the faunistic and floristic componants will differ, but if the environmental factors of the first cycle recur, the plants and animals which characterize the first cycle will recur also.

An increase in salt concentration results in a decrease in the number of species, but a proliferation of individuals. A fall in concentration in a permanent body of water results in a rise in the number of species but represented by relatively few individuals. Also there is a considerable augmentation in the primary productivity. 
Species peculiar to the Camargue are found only in brackish water. Only common species are found in fresh water.

In the opinion of the authors all the observed changes are reversible. None-the-less scientifically based management of the Rhône delta is urgently required; management which will preserve the originality of the Camargue without conflicting with the interests of rice-farming and salt industries.

\section{Die Veränderungen der stehenden Gewässer der Camargue im VERLAUF DER LETZTEN 30 Jahre.}

Auf Grund der Angaben zahlreicher Forscher seit 1935 und eigener Untersuchungen seit 1935 beschreiben die Autoren die Entwicklung einiger Wasserflächen der Camargue. Je nach Ort sind die eingetretenen Veränderungen unter dem Einfluss des Menschen (Einrichtung von Salinen und Reisfeldern) oder des Klimas (wechselnder Niederschlag) entstanden. Die Flora und die Fauna folgen mit Veränderungen, dic sich bei gleichen Milieuveränderungen stets gleichen und voraussagbar sind. Die artenmässige Zusammensetzung von Flora und Fauna hängt vom Jahreszyklus der Salinität und vom permanenten oder temporären Charakter des Gewässers ab.

Die Zunahme der Salzkonzentration bewirkt eine Abnahme der Artenzahl, zeitweise aber auch ein Massenvorkommen einiger Arten. Dic Abnahme der Konzentration wenn sie mit Permanenz Gewässer verbunden ist, hat eine Zunahme der Arten zur Folge, bei der aber die Anzahl der Individuen kleiner wird. Die primäre Produktivität wird beträchtlich erhöht.

Die Arten, die durch Ihre Originalität die Camargue charakterisieren, finden sich nur im Brackwasser. Im Süsswasser leben nur banale Arten.

Die Autoren glauben, dass alle beobachteten Veränderungen reversibel sind. Sie glauben jedoch, dass auf Grund der wissenschaftlichen Erkenntnisse dringend in den Salz und Wasserhaushalt des Deltas eingegriffen werden muss, um ihm seine Originalität zu bewahren. Dies muss auf eine Weise geschehen, die weder den Reisbauern noch den Salinen schadet.

\section{TRAVAUX CITÉS}

Aguesse (P.). 1955. Liste des Cladocères nouveaux pour la Camargue. La Terre et la Vie, 102, : 313-314.

Aguesse (P.). 1956. Quelques considérations sur les Copépodes de Camargue. Vie et Milieu, 7 : 38-42.

Aguesse (P.). 1957. Complément à l'inventaire de la faune invertébrée des eaux camarguaises. La Terre et la Vie, 104 : 241-252.

Aguesse (P.). 1957. La classification des eaux poikilohalines, sa difficulté en Camargue, nouvelle tentative de classification. Vie et Milieu, 8 : 341-363.

Aguesse (P.). 1957. Les effets de la vague de froid de l'hiver 1956 sur lå faune des invertébrés aquatiques de Camargue. La Terre et la Vie, 104 : 198-201. 
Aguesse (P.). 1958. La diversité des milieux aquatiques de Camargue et son influence sur le peuplement en Copépodes, Cladocères et Coléoptères hydrocanthares. Cong. Soc. Savantes : 521-528.

Aguesse (P.). 1960. Complément à l'inventaire de la faune invertébrée des eaux camarguaises. La Terre et la Vie, $107: 132-136$.

Aguesse (P.). 1961. Contribution à l'étude écologique des Zygoptères de Camargue. Thèse, Paris, $156 \mathrm{p}$.

Aguesse (P.). et Bigot (L.). 1959. Les Coléoptères hydrocanthares de Camargue : essai écologique et faunistique. La Terre et la Vie, 116 : 128-148.

Aguesse (P.) et Bigot (L.). 1960. Observations floristiques et faunistiques sur un étang de moyenne Camargue : La Baisse Salée de la Tour du Valat. Vie et Milieu, 8 : 248-307.

Aguesse (P.) et Bigot (L.). 1962. Complément à l'inventaire de la faune camarguaise : les Mollusques terrestres et des eaux douces et saumâtres. La Terre et la Vie, 109 : 82-90.

Aguesse (P.) et Dussart (B.). 1956. Sur quelques Crustacés de Camargue et leur écologie. Vie et Milieu, 7 : 481-520.

Blondel (J.). 1964. L'avifaune nidificatrice des eaux saumâtres cama:guaises en 1962 et 1963. La Terre et la Vie, 131 : 309-330.

Engel (H.). 1957. Ökologisch-faunistische Studien im Rhône-Delta, unter besonderer Berücksichtigung der Mollusken. Bonn. Zool. Beitr., $8: 5-55$.

Hertzog (L.). 1935. Notes faunistiques de Camargue. I. Crustacés. Bull. Soc. Zool. France, $60: 265-282$.

Heunteaux (P.). 1962. L'eau et le sel en Camargue. Position du problème et résultats des premières recherches. La Terre et la Vie, 109 : 11-33.

Hoffman (L.). 1959. Esquisse écologique de la Camargue à l'intention des ornithologistes. Avec contributions de R. LÉvêQue, P. Aguesse et L. Bigot. La Terre et la Vie, $109: 410-413$.

Marazanof (F.). 1963. Cycle annuel des populations des Cladocères et Copépodes du Saint-Seren, de la Baisse Salée, des Relongues et des Cerisières de la Tour du Valat. La Terre et la Vie, 17 : $335-356$.

Marazanof (F.). 1964. Cladocères nouveaux pour la Camargue. La Terre et la Vie, 18 : 379-382.

Marazanof (F.). 1964. Introduction à l'étude écologique des Mollusques des caux douces et saumâtres de Camargue. La Terre et la Vie, 18 : $359-374$.

Marazanof (F.). 1964. Complément à l'inventaire de la faune invertébrée camarguaise : Mollusques des eaux douces et saumâtres. La Terre et la Vie, 18 : 375-379.

Marazanof (F.). 1964. Variations quantitatives des populations d'invertébrés aquatiques de Camargue pendant I'hiver 1962-1963. La Terre et la Vie, 18 : 350-358.

Marazanof (F.). 1965. Ostracodes de Camargue. Ann. Limnol., 1 : 95-102.

Nourisson (M.) et Aguesse (P.). 1961. Cycle annuel des Phyllopodes d'une mare temporaire de Camargue. Bull. Soc. Zool. France, 86 : 754-762. 
Pauıus (M.). 1949. Malacologie maritime et saumâtre de la Camargue. Saintes. 182 p.

Petit (G.) et Schachter (D.). 1954. La Camargue. Etude écologique et faunistique. Année biol., $20: 193-253$.

Petit (G.) et Schachter (D.). 1954. Notes sur l'évolution hydrologique et écologique de l'étang du Vaccarès. La Terre et la Vie, 101 : 121-128.

Schachter (D.). 1944. Contribution à l'étude des Mollusques d'eaux douces et d'eaux saumâtres de la Camargue. Bull. Mus. Hist. Nat. Mar'seille, 4 : 119-134.

Schachter (D.). 1945. Un Copépode nouveau pour la faune française, Diaplomus wierzejskii Richard, en Camargue. Bull. Mus. Hist. Nat. Marseille, 5 : 17-24.

Schachter (D.) . 1950. Contribution à l'étude écologique de la Camargue. Ann. Inst. Océan., $52: 1-108$.

Schachter (D.). 1960. Données nouvelles sur l'évolution de quelques étangs du delta du Rhône. Comm. int. expl. Sci. Mer Médit. Monaco, 15, 3 : 155-162.

Schachter (D.) et Conat (M.). 1951. Note préliminaire sur la faune des Rizières. Bull. Soc. Zool. France, $76: 365-370$.

Tallon (G.). 1938. Les eaux en Camargue au cours de l'année 1937. Bull. Soc. Nat. Accl., Actes de la Réserve, 21 : 11-15.

Tallon (G.). 1949. Observations botaniques. La Terre et la Vie, 96 : 64-67.

Talzon (G.). 1950. Additions à la flore de Camargue. La Terre et la Vie, $97: 265-274$.

Tallon (G.). 1954. Transformation de la Camargue par la riziculture. Evolution du Vaccarès. La Terre et la Vie, 101 : 65-79.

(Ecole Normale Supérieure de Paris, Laboratoire de Zoologie,

Station Biologique de la Tour de Valat, Laboratoire de Zoologie de la Faculté des Sciences, Toulouse.) 


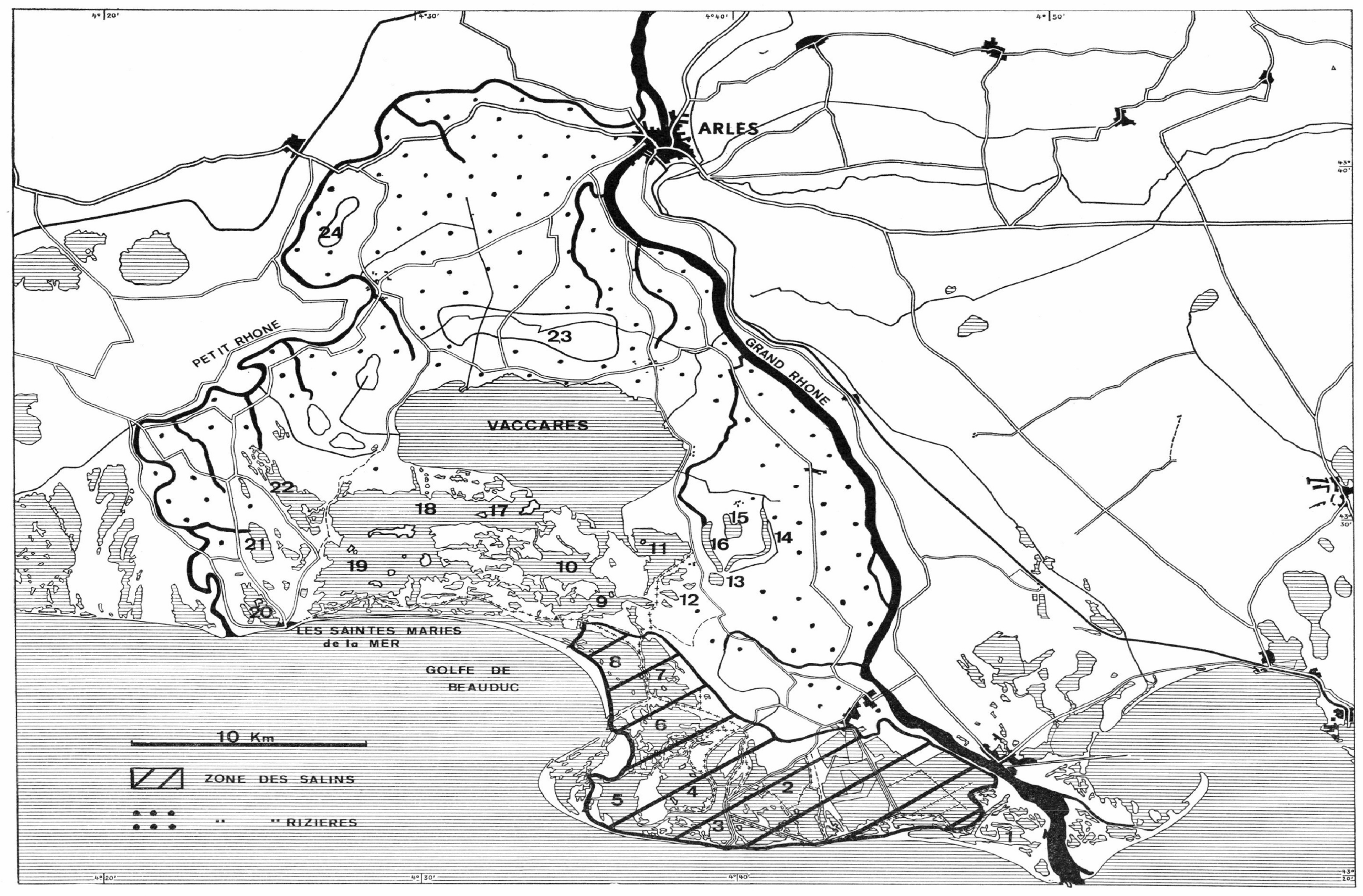

Fig. 3. Carte : Principaux milieux aquatiques de Camargue : 1) Étang de Grande Palun; 2) Étang de Faraman; 3) Vieux-Rhône; 4) Étang du Vaisseau; 5) Etang de Beauduc; 6) Etang Grand-Rascaillan; 7) Etang Fangassier; 8) Etang Galabert; 9) Etang de la Dame; 10) Etang du Lion; 11) Etang
Fournelet; 12) Etang d'Ulmet; 13) Etang Redon; 14) Re'ongues; 15) S aint-Seren; 16) Baisse Salée de la Tour du Valat; 17) Etang de Monro; 18) Etang de Malagroy; 19) Etang des Impériaux; 20) Etang des Launes; 21) Etang de Ginès; 22) Etang de Consécanière; 23) Marais de la Grand Mar, 24) Marais de Saliers. En pointillé, zones de rizières; en trait noir plein, canaux. La partie hachurée correspond à la zone des Salins. 


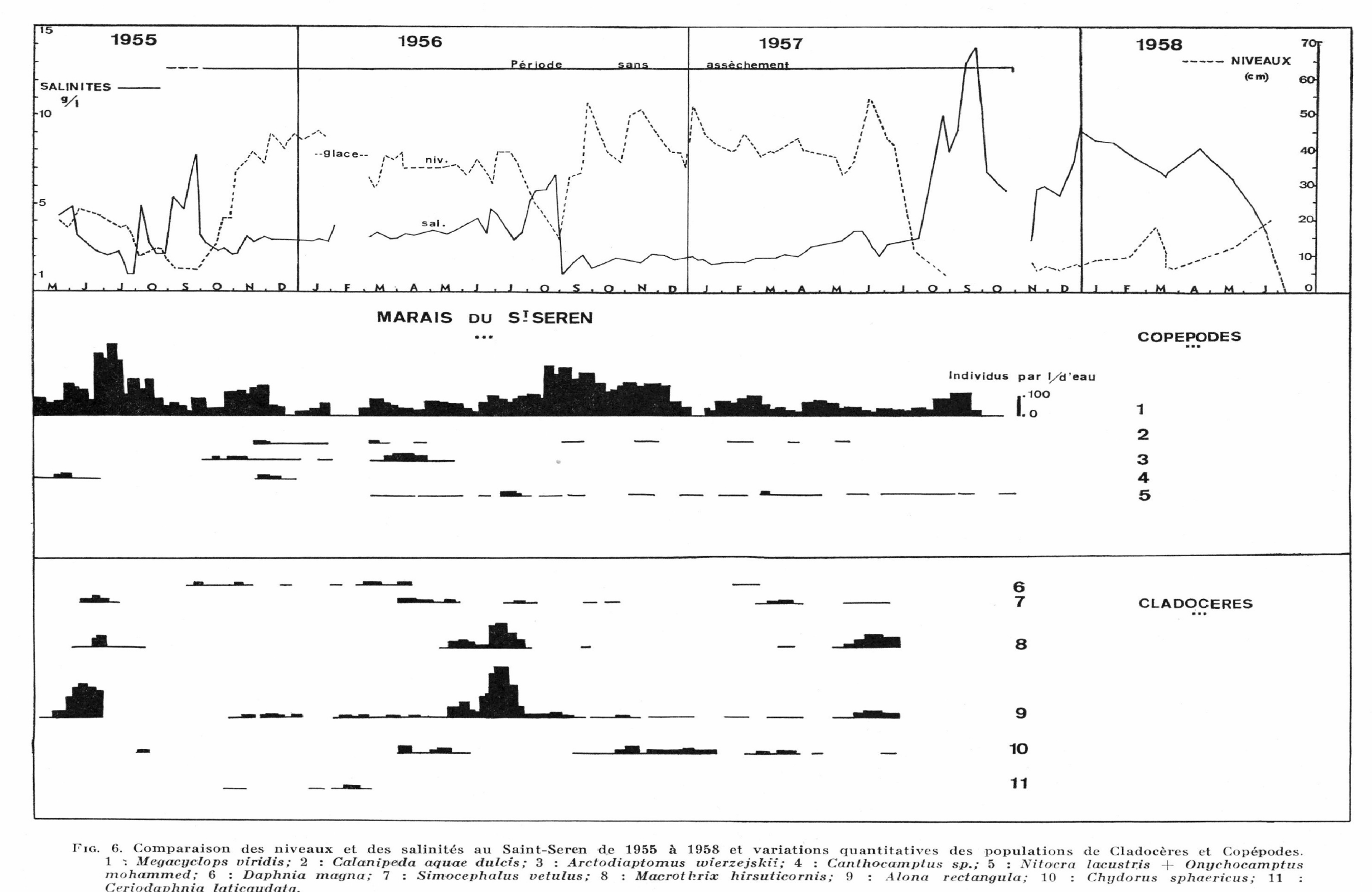

